\title{
Review Article \\ Dwarf Cosmology with the Stromlo Missing Satellites Survey
}

\author{
Helmut Jerjen \\ Research School of Astronomy \& Astrophysics, Mt Stromlo Observatory Australian National University, \\ Cotter Road, Weston ACT 2611, Australia \\ Correspondence should be addressed to Helmut Jerjen, jerjen@mso.anu.edu.au
}

Received 11 May 2009; Accepted 30 July 2009

Academic Editor: Regina Schulte-Ladbeck

Copyright (c) 2010 Helmut Jerjen. This is an open access article distributed under the Creative Commons Attribution License, which permits unrestricted use, distribution, and reproduction in any medium, provided the original work is properly cited.

The standard Lambda Cold Dark Matter model is considered to be a triumph of theoretical astrophysics but observations of the Milky Way and its system of satellite galaxies irresistibly signal that theory is incomplete on galactic and subgalactic scales. The Stromlo Missing Satellites (SMS) Survey is a critical endeavor to investigate at what level predictions of CDM cosmology are consistent with the observed matter distribution in the Milky Way halo. It will be the deepest, most extended search for optically elusive satellite galaxies to date, covering 20000 square degrees of sky. The international SMS Survey collaboration will exploit 150 TB of CCD images in six filters acquired by the new SkyMapper telescope of the Australian National University over the next five years, expecting on completion photometric limits 0.5-1.0 mag fainter than the Sloan Digital Sky Survey. The primary objective of the program is to characterise the baryonic and dark matter components of a complete sample of MW satellites in the Southern hemisphere to provide stringent observational constraints for improving our understanding of how the Milky Way formed and what physical processes governed galaxy formation and evolution in general.

\section{Satellite Galaxies}

According to cosmological theory, overdensities of cold dark matter gravitationally collapsed and formed the first structures in the Universe one billion years after the Big Bang. The gravitational pull of those dark matter clumps drew in primordial baryons in the form of hydrogen gas, providing the seeds for galaxy formation. The observational Universe today is populated with galaxies, the prime repositories of stars, the shining baryonic matter. For obvious reasons, most of the detected and catalogued galaxies are intrinsically the largest and the brightest, those that can be seen from the greatest distance and are most easily studied against the night sky. Ironically, a major limitation on our ability to develop a physically consistent model that describes how galaxies evolved out of the dark matter and baryonic ingredients comes from our incomplete picture of the vicinity of the Milky Way, in particular from the lack of a good understanding of the phenomenon "satellite galaxies." This term is broadly used for dwarf galaxy companions of the Milky Way (Figure 1), some of which contain only a few thousand stars. They cover a stellar mass range of $10^{3-7} \mathcal{M}_{\odot}$ and consist of up to $99.9 \%$ of dark matter (e.g., [1-7]). At the low end of the mass scale, satellites exhibit extreme low star densities so they are completely resolved, that is, transparent, and hard to detect against the screen of MW foreground stars. A few examples of such ultrafaint satellites have been discovered around the Milky Way in the recent years. Cold Dark Matter theory tells us that those are the survivors of the accretion/merger process that assembled larger galaxies. Hence, if we want to shed light on the nature of dark matter and understand how galaxies formed we have to spend a much greater amount of effort on finding and physically characterising these faintest, most elusive galaxies that exist in the Universe.

\section{Cold Dark Matter Theory to the Test}

While the cold dark matter paradigm successfully explains the large-scale distribution of bright baryons (i.e., luminous galaxies) in the Universe (e.g., [8]), one crucial test of the model lies in its ability to predict the properties of dark matter on subgalactic scales, most fundamentally the number and distribution of substructures around the Milky Way and their optical manifestation, the satellite galaxies. 
A generic result of high-resolution numerical simulations of large galaxies [9-12] is that the dark matter halo of the Milky Way must be filled with hundreds or even thousands of smaller dark matter condensations [13]. It is thought that the majority of these subhalos will gravitationally collect sufficient primordial hydrogen gas, turn it into stars, and form MW satellite galaxies observable today. However, the number of bound dark matter subhaloes exceeds the entire population of currently known MW satellites by at least one order of magnitude. This discrepancy is discussed in the literature as the "missing satellites" or "substructure" problem $[9,14]$. There has been no shortage of solutions proposed, ranging from a drastic modification of the dark matter power spectrum [15] or self-interacting dark matter [16] to the reduction of the amount of small-scale features through warm dark matter-like models (e.g., $[17-20]$ ) to careful estimates of the effects of the epoch of photoionization on the ability of subhalos to retain baryons and evolve into luminous satellite galaxies (e.g., [21]).

More recently focus has shifted to the question whether the known Milky Way satellites can actually originate from a population of dark matter subhalos. Even if the suppression of star formation by cosmic reionization and other mechanisms was quite effective in the early Universe and thus the observed satellite galaxies today are comprising only a subset of the actually present CDM substructures, their number-density distribution should be still consistent with an isotropic or mildly triaxial parent distribution. However, Kroupa and collaborators [22-24] showed that the spatial distribution of the luminous Milky Way satellites that was known to be anisotropic for more than 30 years [25-29] is well described by a plane of finite thickness, statistically inconsistent with the distribution of a cosmological dark matter substructure population at the $99.5 \%$ confidence level (see Figure 1). First evidence for a similar disc-of-satellites in M31 has been found by [24] that is now awaiting further testing with the results from the Pan-Andromeda Archaeological Survey (PI McConnachie; http://www.nrc-cnrc.gc.ca/eng/projects/hia/pandas.html).

Although theoretical cosmologists argue that the distribution of the few galaxies in such a "Great Disk of Satellites" almost perpendicular to the MW plane is not improbable (e.g., [30]), debating this issue remains difficult due to the incompleteness of the census of Milky Way satellites, particularly in the crucial parts of the sky away from the proposed plane, regions that are coincidently not covered by the SDSS Survey. Currently, little is known about what physics is concealed behind the "missing satellites" and "Great Disk of Satellites" phenomena but researchers agree that resolving the mystery is going to have a major impact on our understanding of dark matter and its role in the formation process and evolution of galaxies over the lifetime of the Universe.

For example, Kroupa [31] showed that dSph satellite solutions are obtained from ancient tidal dwarf galaxies, implying that the disc-of-satellites can be naturally understood as the remnant of ancient tidal arms that formed during the early assembly of the Milky Way. The almost exact match of one of the author's models with the Hercules satellite [32] is noteworthy. If a significant number of MW $\mathrm{dSphs}$ are confirmed to be of tidal origin this would also imply the substructure crisis to become even more severe. Alternative explanations have been put forward to reconcile this anisotropic distribution within the $\Lambda \mathrm{CDM}$ paradigm. So Li and Helmi [33] and D'Onghia and Lake [34] proposed a scenario where the majority of satellites entered the Milky Way halo in a group rather than individually to explain the spatial and dynamical peculiarities of its satellite distribution. However, no dwarf galaxy associations exist in the vicinity of the Local Group today that are sufficiently compact to produce a disc-of-satellites [35]. On the theoretical side, Libeskind et al. [36] found that one third of their N-body simulations of MW-type halos are in agreement with the observed flattened spatial arrangement and the coherence of angular momenta of the Milky Way satellites. Accurate proper motion estimates for more satellites are needed to properly test the consistency within CDM galaxy formation model.

\section{Searching for the Invisibles}

Because incompleteness in the census of Milky Way satellites prevents any serious scrutiny of the currently best cosmological model, a breakthrough can only be expected once observers established the number and spatial distribution of satellite galaxies around the Milky Way including accurate estimates of those that remain hidden due to detection limits $[37,38]$. Indirect evidence for a large number of undetected Milky Way, satellites was provided by Willman et al. [39]. The comparison of the spatial distributions of satellites around M31, the Milky Way and a simulated galaxy showed that the radial distribution of M31 satellites and the 15 oldest subhaloes of the simulated galaxy are in agreement, while the Milky Way satellites fall below expectations within the limits of observational completeness. Moreover, if one looks at the distribution of satellites as a function of Galactic latitude, the number of Milky Way satellites is less than the simulation predicts at low latitudes.

Northern Hemisphere. Willman et al. [40, 41] conduced a first systematic modern search for Milky Way satellites based on the Sloan Digital Sky Survey (SDSS; [42]) covering 20 percent of the entire sky. The analyses of resolved stars in both the SDSS and the Two Micron All Sky Survey (2MASS) uncovered the Willman 1 and Ursa Major galaxies. Since then, the SDSS collaboration [43-48] and other groups [49, 50] have found already more new Milky Way satellites than were detected in the previous 70 years while the exploitation of the SDSS data is still in process. In an attempt to reach to even lower luminosity systems, Walsh et al. [38, Figure 5] identified a total of 30 ultrafaint candidates in the DR6 footprint (see Figure 2). Establishing the true nature of these stellar overdensities will deeply probe into unknown territory of the galaxy luminosity function.

Southern Hemisphere. This part of the sky remains completely unexplored to faint magnitude levels with modern digital imaging data. The great potential of finding new 


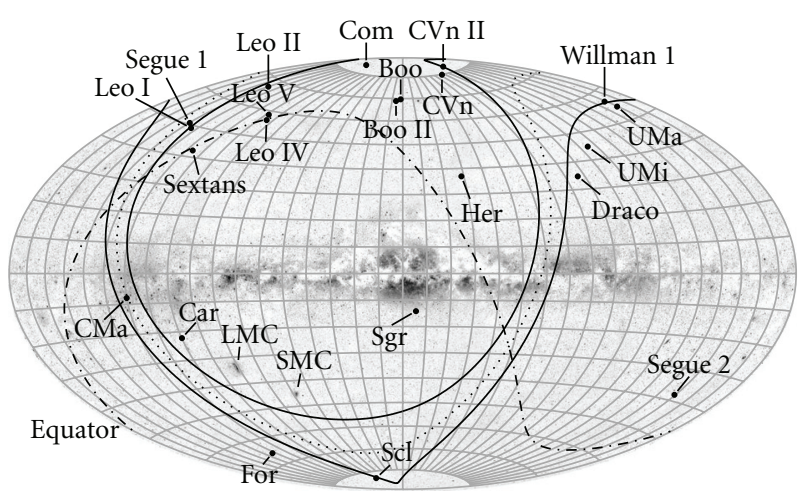

FIgURE 1: The distribution of currently known Milky Way satellites out to the $250 \mathrm{kpc}$ virial radius from the center of the Milky Way. The arrangement of the galaxies in a "Great Disk of Satellites" almost perpendicular to the Galactic plane is highlighted by the dotted line and the arbitrarily chosen \pm 15 degree band. Half of the sky will be searched for satellites by the SMS survey (area south of the celestial equator).

Milky Way satellites in the Southern hemisphere was demonstrated when the analysis of stars from 2MASS led to the discovery of the Canis Major dwarf galaxy (see [51-53]; but see [54] for an update on the controversy of the nature of the CMa phenomenon). Covering twice the area of the SDSS footprint, the Southern hemisphere is expected to conceal at least 30 satellites more luminous than Boo II, Willman 1, or Segue 2.

\section{The Stromlo Missing Satellites Survey}

The Stromlo Missing Satellites (SMS) Survey (see http:// msowww.anu.edu.au/ jerjen/SMS_Survey.html) was initiated to carry out the deepest, most extended search for Milky Way satellites over the next five years, covering the entire 20000 square degrees of the Southern hemisphere. The international SMS Survey collaboration will analyse the digital images obtained by the Australian National University (ANU) SkyMapper Telescope and will use specialized data mining tools on the catalogues produced from the images by the SkyMapper team. Extensive follow-up observations of newly detected satellite candidates are absolutely essential and require the most powerful telescopes to gain an accurate picture of their physical nature. Recent follow-up works on one of the most luminous MW satellites, the CMa dwarf [54, and references therein], and the least luminous Segue $1[55,56]$ highlight some of the many challenges.

4.1. The ANU SkyMapper Telescope. Large CCD arrays are finally becoming available to construct wide-field CCD cameras at optical telescopes. Given this new research window of wide-field astronomy it is not surprising that almost every major observatory worldwide is in the process of developing survey telescopes base on that technology: Pan-STARRS of the University of Hawaii, the near-infrared $4 \mathrm{~m}$ Survey Telescope VISTA of the European Southern Observatory, and on an even larger financial and time scale, the $8.4 \mathrm{~m}$ Large
Synoptic Survey Telescope (http://www.lsst.org/lsst), a widefield telescope facility that is planned to have first light in 2015.

In this latest development, the ANU $1.35 \mathrm{~m}$ SkyMapper telescope (http://www.mso.anu.edu.au/skymapper/) at Siding Spring Observatory represents an investment in Australian frontier technologies of $A \$ 13$ million. It is among the first of this new breed of specialised telescopes capable of scanning the night sky more quickly and sensitively than ever before featuring a $32 \times 4 \mathrm{k} \times 2 \mathrm{k}$ CCD mosaic camera with 0.5 arcsec pixels and a 5.7-sq degree field of view. The primary goal of the facility is to perform the Southern Sky Survey (hereafter S3) over the next five years, producing 150 Terabytes of CCD images and a reference catalogue for one billion stars and galaxies. This program is a six-colour photometry survey based on the SDSS filter set $(u, g, r, i$, and $z)$ plus a Strömgren-like $v$ filter. It will cover all 20000 square degrees south of the celestial equator in 3845 individual pointings with photometric limits 0.51.0 magnitudes fainter than SDSS. The absolute stellar photometry accuracy is expected to be three percent. The S3 is carried out in six full scans of the sky each pointing of 90 seconds duration and a total integration time of 540 seconds. On completion, the $\mathrm{S} 3$ is expected to be 70 times more sensitive than 2MASS and 1.5 times deeper than the northern hemisphere SDSS. A single S3 epoch requires approximately six months to complete. For a detailed description of the SkyMapper observing cadence, the reduction pipeline, photometric and astrometry calibration, and accuracy we refer to Keller et al. [57].

4.2. Needles in the Haystack. The S3 catalogue is the ideal data set to continue the success story of finding new dwarf galaxies in the Milky Way halo. Out to the virial radius of $250 \mathrm{kpc}$, Milky Way satellites are completely resolved into stars. Hence, a satellite initially reveals its presence only through an unusual concentration of faint stars in the sky behind a foreground screen of disk stars, embedded in halo and possibly tidal stream stars, and superimposed on a sea of mid-to-high redshift galaxies. This excess of stars will also populate a well-defined locus in the colour-magnitude diagram depending on the satellite's star formation history (see Figure 3).

In practice, searching for new satellites in the multidimensional parameter space of galactocentric distance, satellite SFH, star density, and angular size is a computer intensive process; the S3 catalogue will contain coordinates and photometric parameters for approximately one billion stars but only about 1:100 000 belong to a satellite galaxy. The SMS team has already a working data mining algorithm in place that was originally developed by Willman et al. [41] and subsequently extended and improved by Walsh et al. [38]. In essence, it is a cluster detection algorithm with capabilities to analyse colour-magnitude diagrams of any given patch of sky. The important preselection of stars in the colour-magnitude diagram employs a comprehensive library of isochrone filters based on Padova evolutionary tracks $[58,59]$. This selection process is repeated to scan through various distance slices. The concept of this search 

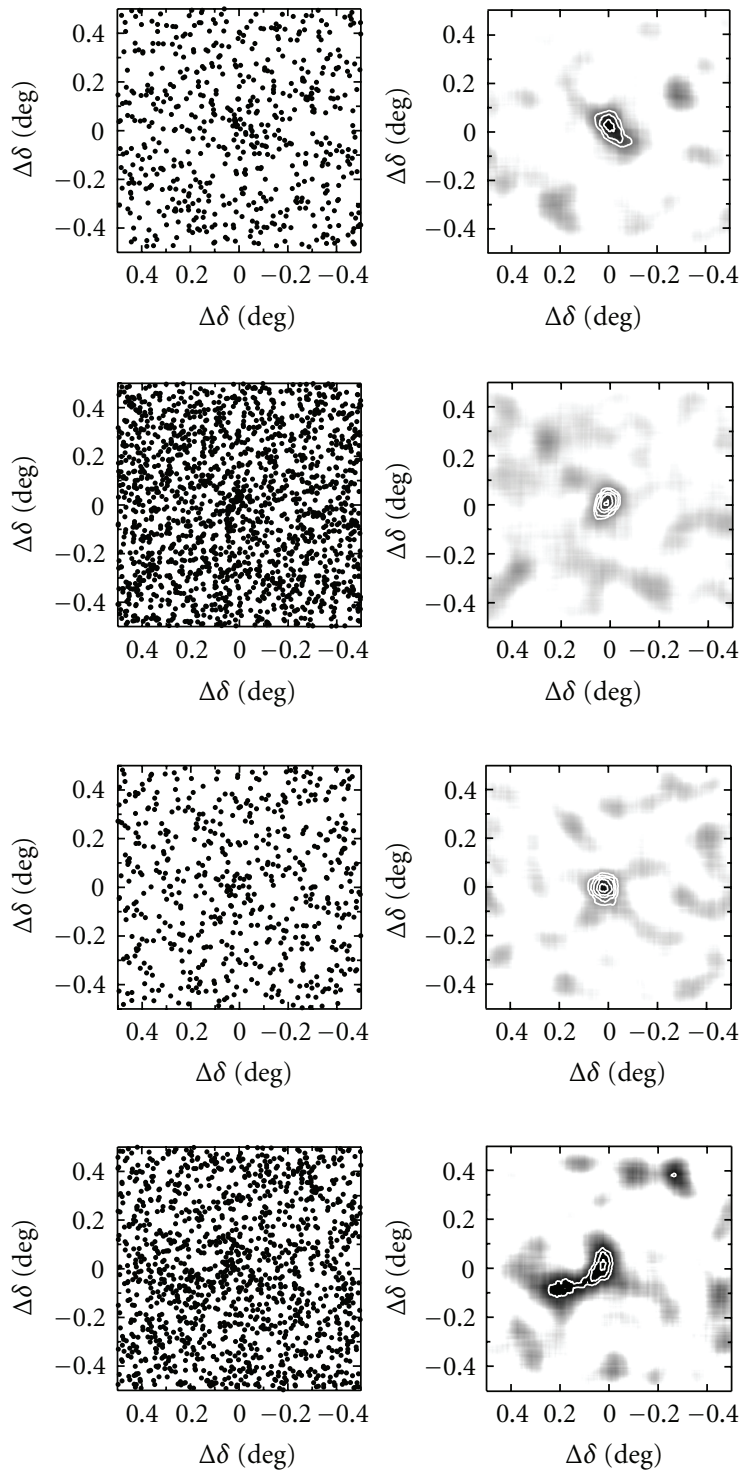
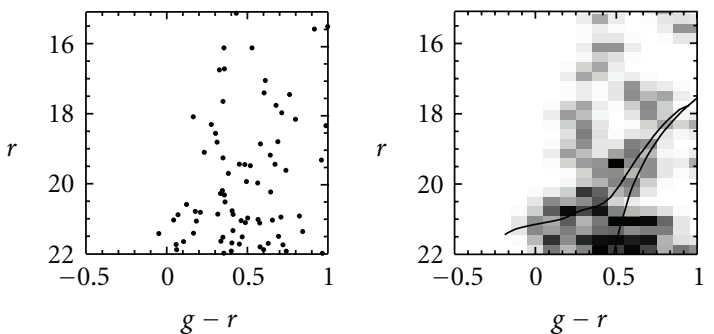

(a)
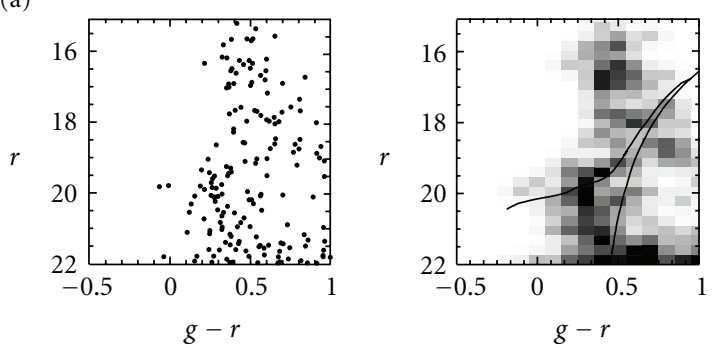

(b)
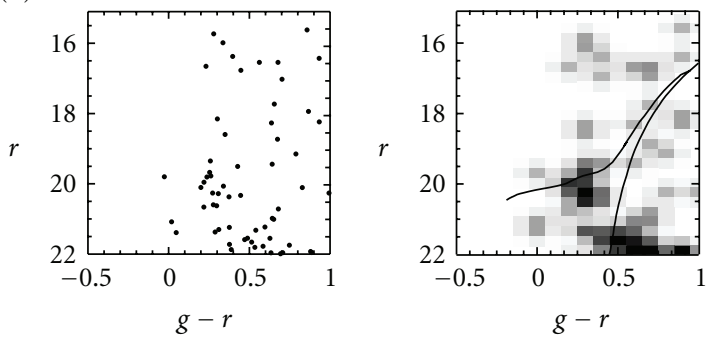

(c)
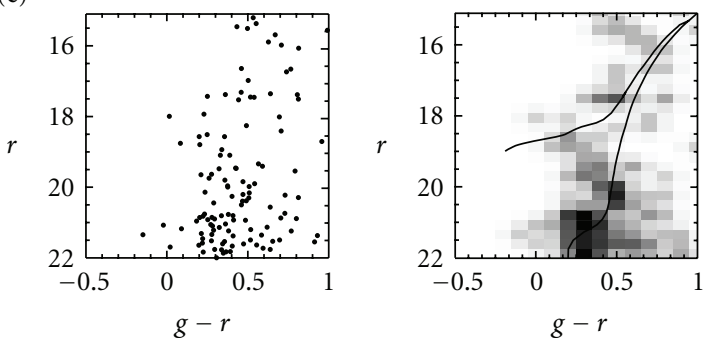

(d)

FIGURE 2: Four stellar overdensities found in SDSS-DR6 with detection significances equal to or greater than known MW satellites. Candidates were found (from top to bottom) in the constellations Canes Venatici, Hercules, Ursa Major, and Virgo. Isochrones in the Hess diagrams (right panel) show at what heliocentric distances those overdensities produced the strongest signal (figures from [38]).

strategy has been extensively tested [38] by taking advantage of the publicly available Sloan Digital Sky Survey data (DR5 and DR6). In single-blind experiments every Northern Hemisphere Milky Way satellite was recovered as well as the previously unknown satellite Boötes II $[50,60]$.

\subsection{Satellites, Light Beacons, or Extreme Globular Clusters.} Stochastic fluctuations in the distribution of MW halo stars are one of the major sources of confusion in the search for satellite candidates. Our algorithm has been carefully calibrated to separate these unwanted sources from true stellar overdensities. Detection thesholds were set above the 1 in 10000 chance for a false positive. However, we cannot exclude a priori the possibility that some of the stellar overdensities turn out to be extremely low luminosity globular clusters, such as Koposov 1 and 2 [61], or stellar condensations in large-scale stellar streams $[45,62,63]$. Any of these alternatives would be equally important to confirm.

Ultrafaint satellites such as Willman 1, Boo II, and Segue 2 with absolute magnitudes $M_{V \text {,tot }} \sim-2.7[48,66]$ are less luminous than the median Milky Way globular cluster. Hence, the expected numbers of RGB and HB stars are naturally small (see Figure 3 ) making it necessary to obtain accurate photometry fainter than the Main Sequence Turnoff (MSTO) to confirm/negate spurious detections to 


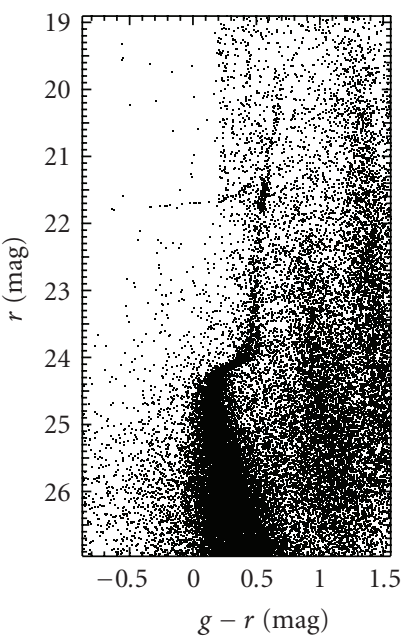

(a)

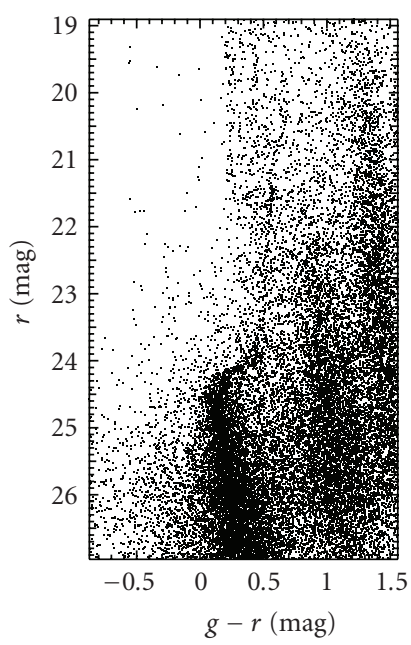

(b)

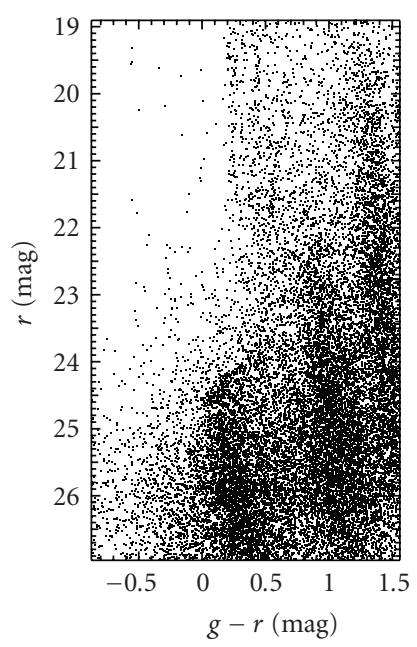

(c)

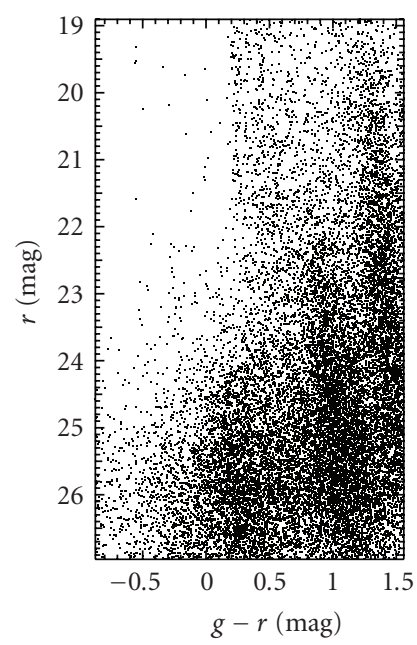

(d)

Figure 3: A series of four $(g-r, r)$ mock colour-magnitude diagrams of a $10 \mathrm{Gyr}$ old, $[\mathrm{Fe} / \mathrm{H}]=-2.0$ single burst stellar population (Padua library) at $150 \mathrm{kpc}$ with realistic Galactic foreground (TRILEGAL; [64]), background galaxies (ACS; [65]) and photometric errors as expected from observations at an 8-m class telescope. Each stellar population has its total luminosity decreased by one magnitude from left to right. The top of the main sequence and the MSTO remain the only visible features that reveal the presence of an ultrafaint dwarf.

the highest possible degree. As a point of reference, the MSTO of a $10 \mathrm{Gyr}$ old, $[\mathrm{Fe} / \mathrm{H}]=-2.0$ stellar population at $150 \mathrm{kpc}$, is at $r \sim 24.2$ magnitude. Ideally, one aims at 1.0 mag below the MSTO $(\mathrm{S} / \mathrm{N} \sim 10)$ in both photometric bands to have a sufficiently large number of stars available for deriving a mean age and metallicity of the population from isochrone fitting and to measure the distance modulus. Deep photometry combined with the fact that these stellar systems have a typical half-light angular size of 10-20 arcmin (see Figure 2) demands a large aperture telescope and an CCD camera with a wide field of view. Imagers like IMACS at the $6.5 \mathrm{~m}$ Magellan telescope $(27 \times$ $27 \mathrm{sq}$ arcmin FOV) or Suprime-Cam at the $8 \mathrm{~m}$ Subaru Telescope $(34 \times 27$ sq arcmin FOV $)$ come immediately to mind.

4.4. Kinematics and Dark Matter Contents. The importance of Milky Way satellites extends far beyond the simple construction of a complete list with spatial coordinates. Stellar velocity measurements over the last 20 years continue to show that the luminous satellites all have central velocity dispersions of the order of $10 \mathrm{~km} \mathrm{~s}^{-1}$ (e.g., Sculptor [67, 68]; Fornax [69]; Carina [70]; Sextans [71]; Ursa Minor [7274]; Draco [73-75]; Leo II [76]). These results support the picture that those stellar systems are completely governed by dark matter - up to $99.9 \%$ of the total mass (e.g., [17]). Consequently, each individual satellite represents a prime laboratory to study dark matter physics in great details.

Once a candidate has been confirmed with deep imaging, red-giant candidates in the satellite will be targeted to the faintest possible limit employing multiobject spectrographs such as AAOmega at the AAT and the Michigan/MIKE Fiber Spectrograph on $6.5 \mathrm{~m}$ Magellan telescope. Measuring routinely radial velocities of stars $(V \lesssim 20.5)$ to $2-3 \mathrm{~km} \mathrm{~s}^{-1}$ precision over the last couple of years enabled not only to measure the systemic velocity of a satellite but also its internal velocity dispersion (dynamical mass) assuming spherical symmetry, dynamic equilibrium, velocity isotropy, and a constant stellar mass-to-light ratio $[4,5,55,77$, and references therein]. After the SMS Survey completion we hope to have measured these quantities for as many as 30 satellites providing a stringent test for the notion that dwarf satellites have a common total mass of $10^{7} \mathcal{M}_{\odot}$ within the central 300 parsec $[7,70,78,79]$.

In cases where sufficient stellar spectra can be measured across the surface of the dwarf satellite, its projected velocity dispersion profile and the underlying mass density profile can also be derived $[39,50]$. These results will allow a statistically robust comparison with the dark matter halo profiles determined from high-resolution N-body simulations to address the core-cusp problem, that observed galactic dark matter haloes show a density profile with a flat core (e.g., $[80,81])$ while CDM haloes have divergent density (a cusp) at the centre $[82,83]$.

As a valuable byproduct of radial velocity measurements the high resolution spectra of the more luminous stars in these dwarf satellites will be very useful for a chemical abundance analysis (e.g., [84-87]). This will enable to test the "galactic building block" hypothesis in which the Milky Way galaxy grew through the merging of smaller dwarf galaxies. Current observations of the four dwarf satellites Sculptor, Sextans, Fornax, and Carina have shown a significantly different chemical composition from stars in the Milky Way halo (e.g., $[88,89])$, leading to the suggestion that not the bright, but rather the newly discovered ultrafaint MW satellites are the predicted building blocks (e.g., [90]). 


\section{Possible Outcomes}

A cosmological model is only as good as it is capable of describing what is observed. On galaxy scales these are the internal and external physical properties of dwarf satellite galaxies. From cold dark matter simulations we expect the virialized extent of the Milky Way's halo (radius $<250 \mathrm{kpc}$ ), which has an almost spherically symmetric shape $[91,92]$ to contain hundreds of subhalos following a power-law radial distribution. After the accomplishment of a full analysis of the SDSS, SkyMapper, and Pan-STARRS data, a highly complete census of MW satellites over the entire sky will be available, based on data mining algorithms that reliably pick up $M_{V}=-5$ dwarfs out to that galactocentric distance. If the number of satellites will be still relatively small and their spatial configuration isotropic, the conclusion would be that they comprise indeed only a subset of the existing lowmass DM halos orbiting the Milky Way [93-97]. The ratio of observed satellites to dark matter subhalo numbers would tell us about the effectiveness of processes such as gas physics, photoionization, and stellar feedback to suppress or evacuate baryons from low mass dark matter halos. First attempts of detailed comparisons between the Milky Way satellite luminosity function down to its faint end and subhalo predictions from $\Lambda$ CDM simulations have been presented over the last year [98-102]. On the one hand, the MW dwarf satellites would be superb targets to discriminate between competing dark matter particle models including the search for annihilation signals (e.g., $[11,103])$ using the upcoming new generation of ground-based Imaging Air Cherenkov Telescopes like MAGIC II and CTA. On the other hand, the reconciliation of CDM theory and observations at that level would prompt the development of innovative techniques to search for the "missing" pure dark CDM substructures in the Milky Way halo (e.g., [104]).

However, if after all the observational effort the sky distribution of any number of genuine satellites is still consistent with a planar or highly anisotropic configuration, the result would be severely at odds with the apparent sphericity of the Milky Way dark matter halo, and even more at odds with an oblate halo having the same orientation as the MW disk. Such a finding would lend strong support to the hypothesis that the majority of MW dwarf satellites have a noncosmological origin and thus lack significant amounts of dark matter. They could have formed from a gas-rich parent satellite on an eccentric near-polar orbit that interacted with the proto-MW [22] or during the dense early stages of the Universe, when the proto-Milky Way and M31 were pulled apart by the general cosmological expansion [105].

In this debate it is interesting to note that the list of the most luminous Milky Way satellites is essentially complete, modulo the possibility of a bright satellite lurking behind the Galactic disk. Accordingly, these "classical" satellites will always show a disk-like arrangement independently of how many low luminous systems will be added to the population in the future. Hence there is a fair chance that the MW dwarf satellites may themselves be divided into two subclasses with different origins. While we look forward in great anticipation to the Stromlo Missing Satellites Survey and similar programs there is no doubt that MW dwarf satellites will play a central role in near-field cosmology for decades to come.

\section{Acknowledgments}

The author thanks both referees for their comments that helped to improve the presentation and the quality of this paper. He also acknowledges the financial support from the Go8-DAAD-Australia/Germany Joint Research Cooperation Scheme and from the Access to Major Research Facilities Programme which is a component of the International Science Linkages established under the Australian Government Innovation Statement, Backing Australia's Ability.

\section{References}

[1] M. Mateo, "Dwarf galaxies of the Local Group," Annual Review of Astronomy and Astrophysics, vol. 36, no. 1, pp. 435506, 1998.

[2] J. T. Kleyna, M. I. Wilkinson, N. Evans, and G. Gilmore, "Ursa Major: a missing low-mass CDM halo?" The Astrophysical Journal, vol. 630, no. 2, pp. L141-L144, 2005.

[3] N. F. Martin, R. A. Ibata, S. C. Chapman, M. Irwin, and G. F. Lewis, "A Keck/DEIMOS spectroscopic survey of faint Galactic satellites: searching for the least massive dwarf galaxies," Monthly Notices of the Royal Astronomical Society, vol. 380, no. 1, pp. 281-300, 2007.

[4] J. D. Simon and M. Geha, "The kinematics of the ultra-faint Milky Way satellites: solving the missing satellite problem," The Astrophysical Journal, vol. 670, no. 1, pp. 313-331, 2007.

[5] M. G. Walker, M. Mateo, E. W. Olszewski, et al., "Velocity dispersion profiles of seven dwarf spheroidal galaxies," The Astrophysical Journal, vol. 667, pp. L53-L56, 2007.

[6] A. Koch, M. I. Wilkinson, J. T. Kleyna, et al., "Stellar kinematics and metallicities in the Leo I dwarf spheroidal galaxy-wide-field implications for galactic evolution," The Astrophysical Journal, vol. 657, no. 1, pp. 241-261, 2007.

[7] L. E. Strigari, J. S. Bullock, M. Kaplinghat, et al., "A common mass scale for satellite galaxies of the Milky Way," Nature, vol. 454, no. 7208, pp. 1096-1097, 2008.

[8] V. Springel, S. D. M. White, A. Jenkins, et al., "Simulations of the formation, evolution and clustering of galaxies and quasars," Nature, vol. 435, no. 7042, pp. 629-636, 2005.

[9] A. Klypin, A. V. Kravtsov, O. Valenzuela, and F. Prada, "Where are the missing galactic satellites?" The Astrophysical Journal, vol. 522, no. 1, pp. 82-92, 1999.

[10] B. Moore, S. Ghigna, F. Governato, et al., "Dark matter substructure within galactic halos," The Astrophysical Journal, vol. 524, no. 1, pp. L19-L22, 1999.

[11] J. Diemand, M. Kuhlen, and P. Madau, "Dark matter substructure and gamma-ray annihilation in the Milky Way halo," The Astrophysical Journal, vol. 657, no. 1, pp. 262-270, 2007.

[12] V. Springel, J. Wang, M. Vogelsberger, et al., "The Aquarius Project: the subhaloes of galactic haloes," Monthly Notices of the Royal Astronomical Society, vol. 391, no. 4, pp. 1685-1711, 2008.

[13] J. Diemand, M. Kuhlen, and P. Madau, "Formation and evolution of galaxy dark matter halos and their substructure," The Astrophysical Journal, vol. 667, no. 2, pp. 859-877, 2007. 
[14] E. D'Onghia and G. Lake, “Cold dark matter's small-scale crisis grows up," The Astrophysical Journal, vol. 612, no. 2, pp. 628-632, 2004.

[15] M. White and R. A. C. Croft, "Suppressing linear power on dwarf galaxy halo scales," The Astrophysical Journal, vol. 539, no. 2, pp. 497-504, 2000.

[16] D. N. Spergel and P. J. Steinhardt, "Observational evidence for self-interacting cold dark matter," Physical Review Letters, vol. 84, no. 17, pp. 3760-3763, 2000.

[17] P. Colin, V. Avila-Reese, and O. Valenzuela, "Substructure and Halo Density Profiles in a Warm Dark Matter Cosmology," The Astrophysical Journal, vol. 542, p. 622, 2000.

[18] P. Bode, J. P. Ostriker, and N. Turok, "Halo formation in warm dark matter models," The Astrophysical Journal, vol. 556, no. 1, pp. 93-107, 2001.

[19] A. R. Zentner and J. S. Bullock, "Halo substructure and the power spectrum," The Astrophysical Journal, vol. 598, no. 1, pp. 49-72, 2003.

[20] M. T. Busha, A. E. Evrard, and F. C. Adams, "The asymptotic form of cosmic structure: small-scale power and accretion history," The Astrophysical Journal, vol. 665, no. 1, pp. 1-13, 2007.

[21] J. S. B. Wyithe and A. Loeb, "Suppression of dwarf galaxy formation by cosmic reionization," Nature, vol. 441, no. 7091, pp. 322-324, 2006.

[22] P. Kroupa, C. Theis, and C. M. Boily, "The great disk of Milky-Way satellites and cosmological sub-structures," Astronomy and Astrophysics, vol. 431, no. 2, pp. 517-521, 2005.

[23] M. Metz, P. Kroupa, and H. Jerjen, "The spatial distribution of the Milky Way and Andromeda satellite galaxies," Monthly Notices of the Royal Astronomical Society, vol. 374, no. 3, pp. 1125-1145, 2007.

[24] M. Metz, P. Kroupa, and H. Jerjen, "Discs of satellites: the new dwarf spheroidals," Monthly Notices of the Royal Astronomical Society, vol. 394, p. 2223, 2009.

[25] W. E. Kunkel and S. Demers, “The Magellanic Plane," Royal Greenwich Observatory Bulletins, vol. 182, p. 241, 1976.

[26] D. Lynden-Bell, "Dwarf galaxies and globular clusters in high velocity hydrogen streams," Monthly Notices of the Royal Astronomical Society, vol. 174, pp. 695-710, 1976.

[27] D. Lynden-Bell, "Internal kinematics and dynamics of galaxies," in Proceedings of the IAU Symposium, vol. 100, p. 89, D. Reidel, Dordrecht, The Netherlands, 1983.

[28] S. R. Majewski, "The Fornax-Leo-Sculptor stream revisited," The Astrophysical Journal, vol. 431, no. 1, pp. L17-L21, 1994.

[29] F. D. A. Hartwick, "The Structure of the Outer Halo of the Galaxy and its Relationship to Nearby Large-Scale Structure," The Astronomical Journal, vol. 119, p. 2248, 2000.

[30] X. Kang, S. Mao, L. Gao, and Y. P. Jing, "Are great disks defined by satellite galaxies in Milky-Way type halos rare in $\Lambda$ CDM?" Astronomy and Astrophysics, vol. 437, no. 2, pp. 383-388, 2005.

[31] P. Kroupa, "Dwarf spheroidal satellite galaxies without dark matter," New Astronomy, vol. 2, no. 2, pp. 139-164, 1997.

[32] M. G. Coleman, J. T. A. de Jong, N. F. Martin, et al., "The elongated structure of the Hercules dwarf spheroidal galaxy from deep Large Binocular Telescope imaging," The Astrophysical Journal, vol. 668, no. 1, pp. L43-L46, 2007.
[33] Y.-S. Li and A. Helmi, "Infall of substructures on to a Milky Way-like dark halo," Monthly Notices of the Royal Astronomical Society, vol. 385, no. 3, pp. 1365-1373, 2008.

[34] E. D'Onghia and G. Lake, "Small Dwarf Galaxies within Larger Dwarfs: Why Some Are Luminous while Most Go Dark," The Astrophysical Journal, vol. 686, p. L61, 2008.

[35] M. Metz, P. Kroupa, C. Theis, G. Hensler, and H. Jerjen, "Did the Milky Way dwarf satellites enter the halo as a group?" The Astrophysical Journal, vol. 697, no. 1, pp. 269-274, 2009.

[36] N. I. Libeskind, C. S. Frenk, S. Cole, A. Jenkins, and J. C. Helly, "How common is the Milky Way-satellite system alignment?" http://arxiv.org/abs/0905.1696.

[37] S. Koposov, V. Belokurov, N. W. Evans, et al., "The luminosity function of the Milky Way satellites," The Astrophysical Journal, vol. 686, no. 1, pp. 279-291, 2008.

[38] S. M. Walsh, B. Willman, and H. Jerjen, "The invisibles: a detection algorithm to trace the faintest Milky Way satellites," Astronomical Journal, vol. 137, no. 1, pp. 450-469, 2009.

[39] B. Willman, F. Governato, J. J. Dalcanton, D. Reed, and T. Quinn, "The observed and predicted spatial distribution of Milky Way satellite galaxies," Monthly Notices of the Royal Astronomical Society, vol. 353, no. 2, pp. 639-646, 2004.

[40] B. Willman, J. J. Dalcanton, D. Martinez-Delgado, et al., "A new Milky Way dwarf galaxy in URSA major," The Astrophysical Journal, vol. 626, no. 2, pp. L85-L88, 2005.

[41] B. Willman, M. R. Blanton, A. A. West, et al., "A new Milky Way companion: unusual globular cluster or extreme dwarf satellite?" Astronomical Journal, vol. 129, no. 6, pp. 26922700, 2005

[42] D. G. York, J. Adelman, J. E. Anderson Jr., et al., "The sloan digital sky survey: technical summary," The Astronomical Journal, vol. 120, p. 1579, 2000.

[43] D. B. Zucker, V. Belokurov, N. W. Evans, et al., "A new Milky Way dwarf satellite in canes venatici," The Astrophysical Journal, vol. 643, no. 2, pp. L103-L106, 2006.

[44] D. B. Zucker, V. Belokurov, N. W. Evans, et al., "A curious Milky Way satellite in Ursa Major," The Astrophysical Journal, vol. 650, no. 1, pp. L41-L44, 2006.

[45] V. Belokurov, D. B. Zucker, N. W. Evans, et al., "The field of streams: sagittarius and its siblings," The Astrophysical Journal, vol. 642, no. 2, pp. L137-L140, 2006.

[46] V. Belokurov, D. B. Zucker, N. W. Evans, et al., "Cats and dogs, hair and a hero: a quintet of new Milky Way companions," The Astrophysical Journal, vol. 654, no. 2, pp. 897-906, 2007.

[47] V. Belokurov, M. G. Walker, N. W. Evans, et al., "Leo V: a companion of a companion of the Milky Way galaxy?" The Astrophysical Journal, vol. 686, no. 2, pp. L83-L86, 2008.

[48] V. Belokurov, M. G. Walker, N. W. Evans, et al., "The discovery of Segue 2: a prototype of the population of satellites of satellites," Monthly Notices of the Royal Astronomical Society, vol. 397, no. 4, pp. 1748-1755, 2009.

[49] T. Sakamoto and T. Hasegawa, "Discovery of a faint old stellar system at $150 \mathrm{kpc,"} \mathrm{The} \mathrm{Astrophysical} \mathrm{Journal,} \mathrm{vol.} \mathrm{653,} \mathrm{no.} \mathrm{1,}$ pp. L29-L32, 2006.

[50] S. Walsh, H. Jerjen, and B. Willman, "A Pair of Bootes: A New Milky Way Satellite," The Astrophysical Journal, vol. 83, p. L662, 2007.

[51] N. F. Martin, R. A. Ibata, M. Bellazzini, M. J. Irwin, G. F. Lewis, and W. Dehnen, "A dwarf galaxy remnant in Canis Major: the fossil of an in-plane accretion on to the Milky Way," Monthly Notices of the Royal Astronomical Society, vol. 348, no. 1, pp. 12-23, 2004. 
[52] N. F. Martin, R. A. Ibata, B. C. Conn, G. F. Lewis, M. Bellazzini, and M. J. Irwin, "A radial velocity survey of low Galactic latitude structures: I. Kinematics of the Canis Major dwarf galaxy," Monthly Notices of the Royal Astronomical Society, vol. 362, no. 3, pp. 906-914, 2005.

[53] M. Bellazzini, R. Ibata, N. Martin, G. F. Lewis, B. Conn, and M. J. Irwin, "The core of the Canis Major galaxy as traced by red clump stars," Monthly Notices of the Royal Astronomical Society, vol. 366, no. 3, pp. 865-883, 2006.

[54] C. Mateu, A. K. Vivas, R. Zinn, L. R. Miller, and C. Abad, "No excess of RR lyrae stars in the Canis Major overdensity," The Astronomical Journal, vol. 137, pp. 4412-4423, 2009.

[55] M. Geha, B. Willman, J. D. Simon, et al., "The least luminous galaxy: spectroscopy of the Milky Way satellite segue 1," The Astrophysical Journal, vol. 692, pp. 1464-1475, 2009.

[56] M. Niederste-Ostholt, V. Belokurov, N. W. Evans, G. Gilmore, R. F. G. Wyse, and J. E. Norris, "The origin of segue 1," Monthly Notices of the Royal Astronomical Society, vol. 398, no. 4, pp. 1771-1781, 2009.

[57] S. C. Keller, B. P. Schmidt, M. S. Bessell, et al., "The SkyMapper telescope and the southern sky survey," Publications of the Astronomical Society of Australia, vol. 24, no. 1, pp. 1-12, 2007.

[58] L. Girardi, G. Bertelli, A. Bressan, et al., "Theoretical isochrones in several photometric systems I. JohnsonCousins-Glass, HST/WFPC2, HST/NICMOS, Washington, and ESO imaging survey filter sets," Astronomy and Astrophysics, vol. 391, no. 1, pp. 195-212, 2002.

[59] P. Marigo, L. Girardi, A. Bressan, M. A. T. Groenewegen, L. Silva, and G. L. Granato, "Evolution of asymptotic giant branch stars: II. Optical to far-infrared isochrones with improved TP-AGB models," Astronomy and Astrophysics, vol. 482, no. 3, pp. 883-905, 2008.

[60] S. M. Walsh, B. Willman, D. Sand, et al., "Boötes II reBoöted: an MMT/Megacam study of an ultrafaint Milky Way satellite," The Astrophysical Journal, vol. 688, no. 1, pp. 245-253, 2008.

[61] S. Koposov, J. T. A. de Jong, V. Belokurov, et al., "The discovery of two extremely low luminosity Milky Way globular clusters," The Astrophysical Journal, vol. 669, no. 1, pp. 337-342, 2007.

[62] D. Martínez-Delgado, A. Aparicio, M. A. Gómez-Flechoso, and R. Carrera, "Tidal streams in the galactic halo: evidence for the sagittarius northern stream or traces of a new nearby dwarf galaxy," The Astrophysical Journal, vol. 549, no. 2, pp. L199-L202, 2001.

[63] C. J. Grillmair, "Four new stellar debris streams in the galactic halo," The Astrophysical Journal, vol. 693, pp. 1118-1127, 2009.

[64] L. Girardi, M. A. T. Groenewegen, E. Hatziminaoglou, and L. da Costa, "Star counts in the galaxy simulating from very deep to very shallow photometric surveys with the TRILEGAL code," Astronomy and Astrophysics, vol. 436, no. 3, pp. 895-915, 2005.

[65] D. Harsono and R. De Propris, "The luminosity function of galaxies to $M_{B g V r i z} \sim-14$ in $z \sim 0.3$ Clusters," Astronomical Journal, vol. 137, no. 2, pp. 3091-3099, 2009.

[66] N. F. Martin, J. T. A. de Jong, and H.-W. Rix, "A comprehensive maximum likelihood analysis of the structural properties of faint Milky Way satellites," The Astrophysical Journal, vol. 684, no. 2, pp. 1075-1092, 2008.

[67] M. Aaronson and E. W. Olszewski, "Accurate radial velocities for carbon stars in the Sculptor dwarf spheroidal," The Astrophysical Journal, vol. 94, p. 657, 1987.
[68] D. Queloz, P. Dubath, and L. Pasquini, "A kinematic study of the Sculptor dwarf spheroidal galaxy," Astronomy and Astrophysics, vol. 300, p. 31, 1995.

[69] M. Mateo, E. Olszewski, D. L. Welch, P. Fischer, and W. Kunkel, "A kinematic study of the fornax dwarf spheroidal galaxy," Astronomical Journal, vol. 102, no. 3, pp. 914-926, 1991.

[70] M. Mateo, E. W. Olszewski, C. Pryor, D. L. Welch, and P. Fischer, "The Carina dwarf spheroidal galaxy: how dark is it?" Astronomical Journal, vol. 105, no. 2, pp. 510-526, 1993.

[71] N. B. Suntzeff, M. Mateo, D. M. Terndrup, E. W. Olszewski, D. Geisler, and W. Weller, "Spectroscopy of giants in the Sextans dwarf spheroidal galaxy," The Astrophysical Journal, vol. 418, no. 1, pp. 208-228, 1993.

[72] J. C. Hargreaves, G. Gilmore, M. J. Irwin, and D. Carter, "A dynamical study of the Ursa-Minor dwarf spheroidal galaxy," Monthly Notices of the Royal Astronomical Society, vol. 271, p. 693, 1994.

[73] T. E. Armandroff, E. W. Olszewski, and C. Pryor, "The massto-light ratios of the draco and ursa minor dwarf spheroidal galaxies. I. Radial velocities from multifiber spectroscopy," Astronomical Journal, vol. 110, no. 5, pp. 2131-2165, 1995.

[74] E. W. Olszewski, M. Aaronson, and J. M. Hill, "Nine seasons of velocity measurements in the Draco and Ursa Minor dwarf spheroidal galaxies with the MMT echelle," Astronomical Journal, vol. 110, p. 2120, 1995.

[75] J. C. Hargreaves, G. Gilmore, and J. D. Annan, "The influence of binary stars on dwarf spheroidal galaxy kinematics," Monthly Notices of the Royal Astronomical Society, vol. 279, no. 1, pp. 108-120, 1996.

[76] S. S. Vogt, M. Mateo, E. W. Olszewski, and M. J. Keane, "Internal kinematics of the Leo II dwarf spheroidal galaxy," Astronomical Journal, vol. 109, no. 1, pp. 151-163, 1995.

[77] M. Mateo, E. W. Olszewski, S. S. Vogt, and M. J. Keane, "The internal kinematics of the Leo I dwarf spheroidal galaxy: dark matter at the fringe of the Milky Way," Astronomical Journal, vol. 116, no. 5, pp. 2315-2327, 1998.

[78] G. Gilmore, M. I. Wilkinson, R. F. G. Wyse, et al., "The observed properties of dark matter on small spatial scales," The Astrophysical Journal, vol. 663, no. 2, pp. 948-959, 2007.

[79] J. Peñarrubia, A. W. McConnachie, and J. F. Navarro, "The cold dark matter halos of local group dwarf spheroidals," The Astrophysical Journal, vol. 672, no. 2, pp. 904-913, 2008.

[80] W. J. G. de Blok, S. S. McGaugh, A. Bosma, and V. C. Rubin, "Mass density profiles of low surface brightness galaxies," The Astrophysical Journal, vol. 552, no. 1, pp. L23-L26, 2001.

[81] G. Gentile, A. Burkert, P. Salucci, and U. Klein, "The dwarf galaxy DDO 47 as a dark matter laboratory: testing cusps hiding in triaxial halos," The Astrophysical Journal, vol. 634, no. 2, pp. L145-L148, 2005.

[82] J. F. Navarro, C. S. Frenk, and S. D. M. White, "A universal density profile from hierarchical clustering," The Astrophysical Journal, vol. 490, no. 2, pp. 493-508, 1997.

[83] J. F. Navarro, E. Hayashi, C. Power, et al., "The inner structure of $\Lambda$ CDM haloes: III. Universality and asymptotic slopes," Monthly Notices of the Royal Astronomical Society, vol. 349, no. 3, pp. 1039-1051, 2004.

[84] A. Koch, E. K. Grebel, R. F. G. Wyse, et al., "Complexity on small scales: the metallicity distribution of the Carina dwarf spheroidal galaxy," Astronomical Journal, vol. 131, no. 2, pp. 895-911, 2006. 
[85] A. Koch, A. McWilliam, E. K. Grebel, D. B. Zucker, and V. Belokurov, "The highly unusual chemical composition of the hercules dwarf spheroidal galaxy," The Astrophysical Journal, vol. 688, pp. L13-L16, 2008.

[86] E. N. Kirby, P. Guhathakurta, and C. Sneden, "Metallicity and alpha-element abundance measurement in red giant stars from medium-resolution spectra," The Astrophysical Journal, vol. 682, no. 2, pp. 1217-1233, 2008.

[87] J. E. Norris, G. Gilmore, R. F. G. Wyse, et al., "The abundance spread in the boötes I dwarf spheroidal galaxy," The Astrophysical Journal, vol. 689, no. 2, pp. L113-L116, 2008.

[88] A. Helmi, M. J. Irwin, E. Tolstoy, et al., "A new view of the dwarf spheroidal satellites of the Milky Way from VLT flames: where are the very metal-poor stars?" The Astrophysical Journal, vol. 651, no. 2, pp. L121-L124, 2006.

[89] W. Aoki, N. Arimoto, K. Sadakane, et al., "Chemical composition of extremely metal-poor stars in the sextans dwarf spheroidal galaxy," Astronomy \& Astrophysics, vol. 502, no. 2, pp. 569-578, 2009.

[90] E. N. Kirby, J. D. Simon, M. Geha, P. Guhathakurta, and A. Frebel, "Uncovering extremely metal-poor stars in the Milky Way's ultrafaint dwarf spheroidal satellite galaxies," The Astrophysical Journal, vol. 685, no. 1, pp. L43-L46, 2008.

[91] R. P. Olling and M. R. Merrifield, "Two measures of the shape of the dark halo of the Milky Way," Monthly Notices of the Royal Astronomical Society, vol. 311, no. 2, pp. 361-369, 2000.

[92] S. Kazantzidis, A. V. Kravtsov, A. R. Zentner, B. Allgood, D. Nagai, and B. Moore, "The effect of gas cooling on the shapes of dark matter halos," The Astrophysical Journal, vol. 611, no. 2, pp. L73-L76, 2004.

[93] F. Stoehr, S. D. M. White, G. Tormen, and V. Springel, "The satellite population of the Milky Way in a $\Lambda$ CDM universe," Monthly Notices of the Royal Astronomical Society, vol. 335, no. 4, pp. L84-L88, 2002.

[94] E. Hayashi, J. F. Navarro, J. E. Taylor, J. Stadel, and T. Quinn, "The structural evolution of substructure," The Astrophysical Journal, vol. 584, no. 2, pp. 541-558, 2003.

[95] J. S. Bullock, A. V. Kravtsov, and D. H. Weinberg, "Reionization and the abundance of galactic satellites," The Astrophysical Journal, vol. 539, no. 2, pp. 517-521, 2000.

[96] H. Susa and M. Umemura, "Formation of dwarf galaxies during the cosmic reionization," The Astrophysical Journal, vol. 600, no. 1, pp. 1-16, 2004.

[97] A. V. Kravtsov, O. Y. Gnedin, and A. A. Klypin, "The tumultuous lives of galactic dwarfs and the missing satellites problem," The Astrophysical Journal, vol. 609, no. 2, pp. 482497, 2004.

[98] P. Madau, M. Kuhlen, J. Diemand, et al., "Fossil remnants of reionization in the halo of the Milky Way," The Astrophysical Journal, vol. 689, no. 1, pp. L41-L44, 2008.

[99] A. V. Macciò, X. Kang, and B. Moore, "Central mass and luminosity of Milky Way satellites in the $\Lambda$ cold dark matter model," The Astrophysical Journal, vol. 692, pp. L109-L112, 2009.

[100] A. V. Macciò, X. Kang, F. Fontanot, R. S. Somerville, S. E. Koposov, and P. Monaco, "On the origin and properties of Ultrafaint Milky Way Satellites in a LCDM Universe," submitted to Monthly Notices of the Royal Astronomical Society.

[101] Y.-S. Li, A. Helmi, G. De Lucia, and F. Stoehr, "On the common mass scale of the Milky Way satellites," Monthly Notices of the Royal Astronomical Society, vol. 397, p. L87, 2009.
[102] S. E. Koposov, J. Yoo, H.-W. Rix, D. H. Weinberg, A. V. Macciò, and J. M. Escudé, "A quantitative explanation of the observed population of Milky Way satellite galaxies," The Astrophysical Journal, vol. 696, pp. 2179-2194, 2009.

[103] T. Bringmann, M. Doro, and M. Fornasa, "Dark matter signals from Draco and Willman 1: prospects for MAGIC II and CTA," Journal of Cosmology and Astroparticle Physics, vol. 1, p. 16, 2009.

[104] L. V. E. Koopmans, "Gravitational imaging of cold dark matter substructures," Monthly Notices of the Royal Astronomical Society, vol. 363, no. 4, pp. 1136-1144, 2005.

[105] T. Sawa and M. Fujimoto, "A dynamical model for the orbit of the andromeda galaxy M31 and the origin of the local group of galaxies," Publication of the Astronomical Society of Japan, vol. 57, no. 3, pp. 429-446, 2005. 

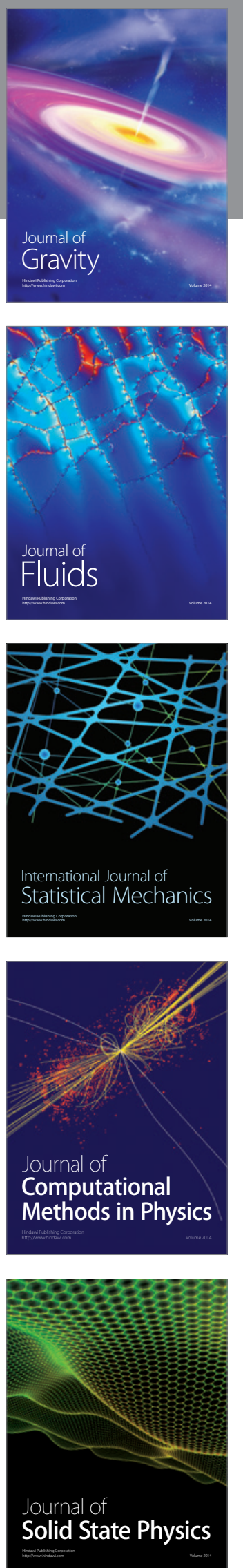

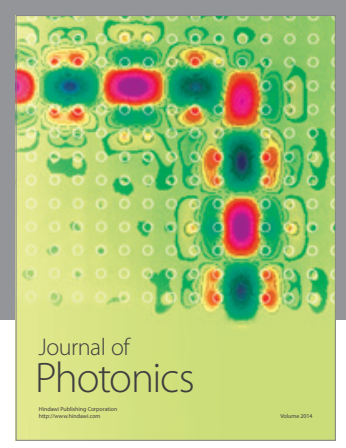

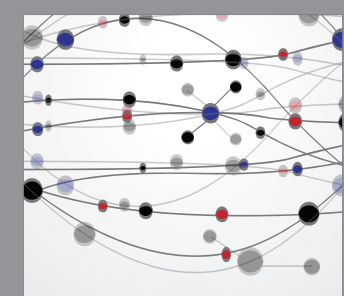

The Scientific World Journal
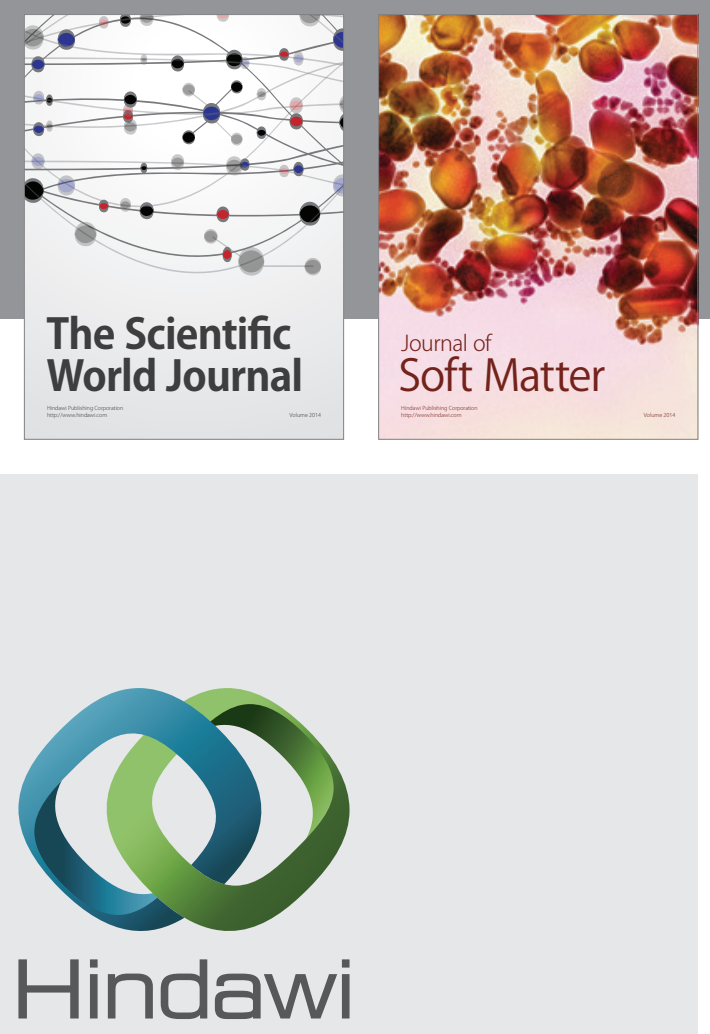

Submit your manuscripts at

http://www.hindawi.com
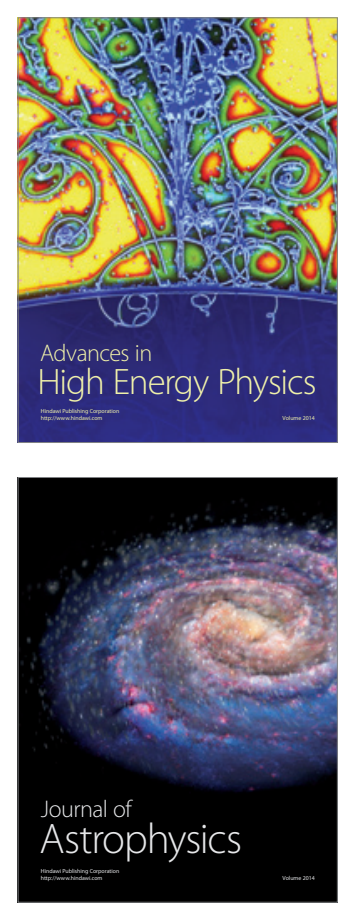
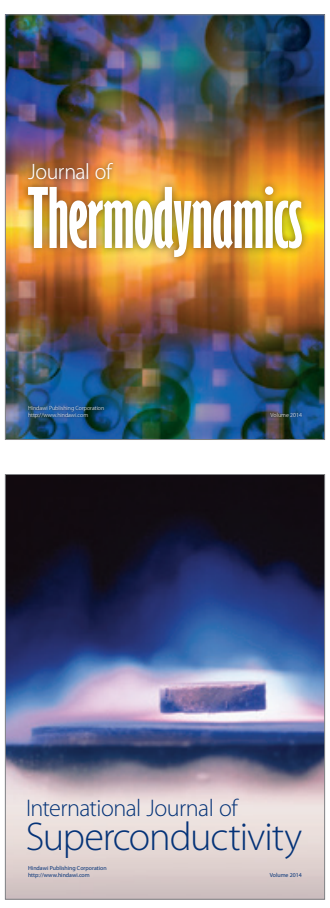
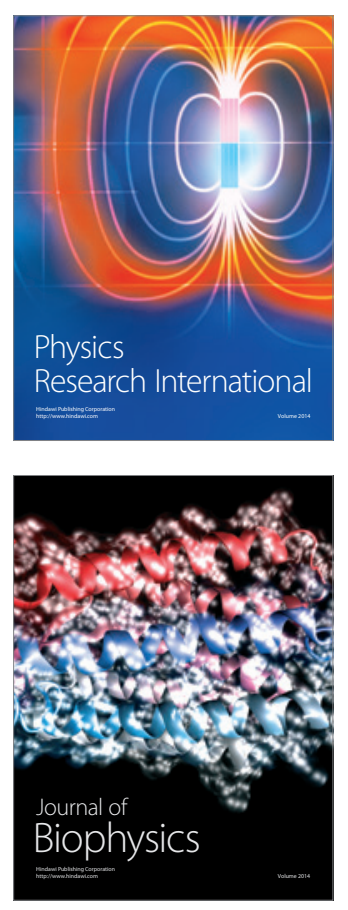
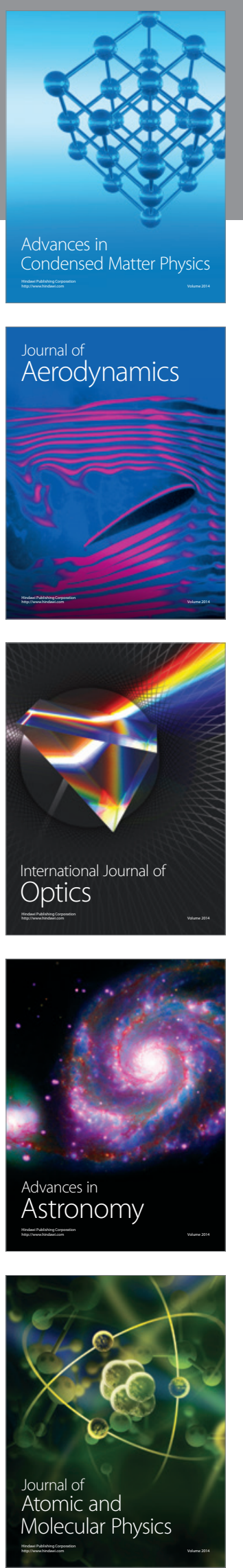